\section{ATU-05 EXPERIENCE OF ENDOSCOPIC SUBMUCOSAL DISSECTION (ESD) FOR TREATMENT OF GASTROINTESTINAL NEUROENDOCRINE TUMORS (NETS)}

${ }^{1}$ Deborah Costa*, 'Alberto Murino, ${ }^{2}$ Ana-Maria Bucalau, ${ }^{3}$ Laurine Verset, ${ }^{2}$ Vincent Huberty, ${ }^{1}$ Andrea Telese, ${ }^{1}$ Nikolaos Lazaridis, ${ }^{4}$ Tu Vinh Luong, ${ }^{5}$ Christos Toumpanakis, ${ }^{5}$ Martyn Caplin, ${ }^{2}$ Jacques Devière, ${ }^{2}$ Arnaud Lemmers, ${ }^{1}$ Edward J Despott. ${ }^{1}$ Royal Free Unit for Endoscopy, The Royal Free Hospital, University College London Institute for Liver and Digestive Health, London, UK; '2Erasme Hospital, Department of Gastroenterology, Hepatopancreatology and Digestive Oncology, Brussels, Belgium; ${ }^{3}$ Erasme Hospital, department of Pathology, Brussels, Belgium; ${ }^{4}$ Department of Cellular Pathology, Royal Free Hospital NHS Foundation Trust, London, UK; ${ }^{5}$ Neuroendocrine Tumour Unit, Centre for Gastroenterology, ENETS Centre of Excellence, Royal Free London NHS Foundation Trust, London, UK

\subsection{6/gutjnl-2019-BSGAbstracts. 18}

Introduction Although endoscopic submucosal dissection (ESD) of gastrointestinal (GI) neuroendocrine tumor (NETs) is an increasingly performed minimally invasive technique allowing complete en-bloc resection of mucosal and submucosal lesions, efficacy and safety outcome is limited. The aim of this study is to review two European centers experience of endoscopic treatment of superficial GI NET by ESD.

Methods Clinical and technical data of patients treated by ESD from two tertiary European centers were prospectively collected from October 2014 to February 2019. Complete resection (R0) was defined as clear lateral and vertical margins.

Results Twenty-four ESDs of NET were performed in 22 patients (females 59\%), mean age of 54.8 years. Fifteen NETs $(66 \%)$ were removed from the stomach, 6 from the rectum (25\%), 1 from the esophagus (4\%) and 1 from the duodenum (4\%). $87.5 \%$ of gastric NETs were associated with atrophic gastritis (86.6\% of which of autoimmune etiology) and 56\% with previous history of multiples NETs. En-bloc resection was achieved in all patients (100\%). R0 resection rate was $75 \%$ (91.6\% clear lateral and 79\% clear vertical margins). Lymphovascular infiltration was seen in one case (4\%).

Median ESD duration time was $77 \mathrm{~min}$ (20-240). Two cases presented small perforations, treated conservatively by antibiotics and clip closure. The median specimen size was of $25 \mathrm{~mm}$ (12-50). Pathological examination showed 58.3\% grade $1,37.5 \%$ grade 2 and $4 \%$ grade 3 NETs. Sixteen lesions were characterized as pT1 (72.7\%). Three patients were candidates for additional treatment: one had endoscopic mucosal resection for additional known lesions, one underwent surgery with oncological lymph node resection (finally pT2N1) and one refused systemic therapy. At the end of a median follow-up of 18 months, two cases of recurrence were identified: one was managed endoscopically, the second refused treatment.

Conclusions Our series of ESD for selected GI NETs showed favorable results in term of efficacy and safety. However, further studies are needed to determine the role of ESD compared to other resection modalities.

\section{AWE-01 FEASIBILITY AND ACCEPTABILITY OF NOVEL COLONOSCOPY WITH COMPUTER AIDED EARLY DIAGNOSIS OF BOWEL CANCER}

${ }^{1}$ Sunil Dolwani*, ${ }^{2}$ Xujiong Ye, ${ }^{1}$ Katherine Brain, ${ }^{3}$ Robert William Howe, ${ }^{4}$ Vladimir Gubala, ${ }^{5}$ Gemma-Louise Davies, ${ }^{1}$ Stephanie Smits. ${ }^{1}$ Cardiff University, Cardiff, UK; ${ }^{2}$ University of Lincoln, Lincoln, UK; ${ }^{3}$ Lostwithiel Medical Practice, Cornwall, UK; ${ }^{4}$ University of Kent, Medway School of Pharmacy, UK; ${ }^{5}$ University College London, London, UK

\subsection{6/gutjnl-2019-BSGAbstracts. 19}

Introduction Patients with low risk bowel symptoms often triaged to a non-urgent pathway have longer diagnostic intervals and later detection of cancer. Resource and workforce constraints in delivery of secondary care colonoscopy contribute to these delays. This study presents preliminary data to investigate the acceptability and feasibility of a novel type of semiautomated robotic colonoscopy (portable with disposable probes not requiring reprocessing or decontamination) to potentially overcome delays between presentation, referral, diagnosis and treatment decision.

Methods Participants referred by their GP for direct colonoscopy underwent the new procedure (semi-automated robotic colonoscopy) and a parallel (back to back design) standard colonoscopy and were later interviewed via telephone to explore their experience and comparison between the two procedures. Comfort scores and colonoscopy quality indicators and outcomes were compared. An online GP survey exploring feasibility of the new procedure in a primary care setting was also conducted. We also analysed the feasibility and accuracy of developing a machine learning tool to interpret findings at both novel and standard colonoscopy.

Results Participants (6 M, 9 F) perceived the value, usefulness and purpose of the new procedure, with favourable comparisons made to standard colonoscopy, particularly previous experience of pain and recovery. Lack of requirement for sedation was a key benefit of the new procedure. Primary care was endorsed as a location, with proximity and familiarity viewed favourably, particularly in overcoming negative aspects of bowel preparation and recovery (e.g. not wanting to travel far). Those feeling they would not need sedation also viewed primary care positively. Others were indifferent, with feelings that it was such an undesirable procedure that location would not matter. Twenty-nine GPs completed the online survey. Barriers to the new procedure in GP practices included perceived lack of expertise to interpret findings (74\% agreement) and not enough space for equipment (59\% agreement). Nearly half of participants felt the new procedure had the potential to impact quicker diagnosis (48\% agreement), and all participants felt it had the potential to achieve early diagnosis in low risk bowel symptoms not meeting 2 week wait criteria (100\% agreement).

Conclusions Target users found the new procedure acceptable and viewed it favourably compared to standard colonoscopy. GPs perceived uses for the new procedure in primary care, particularly for low risk patients, however expertise and space were a concern. These findings are positive as the new 